1

Dielectric spectroscopy for monitoring human pancreatic islet differentiation within cell-seeded scaffolds in a perfusion bioreactor system

J. Daoud, K. Heileman, S. Shapka, L. Rosenberg and M. Tabrizian*

Herein, we describe a platform for dielectric spectroscopy monitoring of scaffold-embedded human pancreatic islet
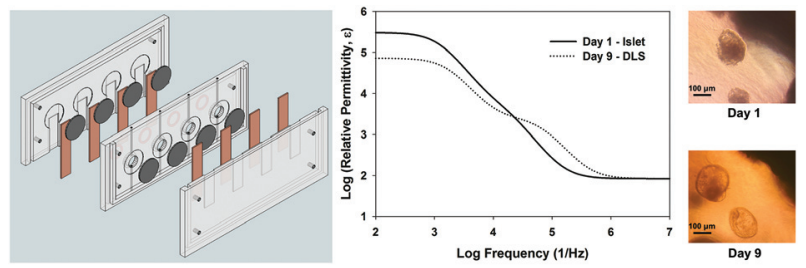
differentiation within a perfusion bioreactor.

Please check this proof carefully. Our staff will not read it in detail after you have returned it.

Translation errors between word-processor files and typesetting systems can occur so the whole proof needs to be read. Please pay particular attention to: tabulated material; equations; numerical data; figures and graphics; and references. If you have not already indicated the corresponding author(s) please mark their name(s) with an asterisk. Please e-mail a list of corrections or the PDF with electronic notes attached - do not change the text within the PDF file or send a revised manuscript. Corrections at this stage should be minor and not involve extensive changes. All corrections must be sent at the same time.

Please bear in mind that minor layout improvements, e.g. in line breaking, table widths and graphic placement, are routinely applied to the final version.

We will publish articles on the web as soon as possible after receiving your corrections; no late corrections will be made.

Please return your final corrections, where possible within $\mathbf{4 8}$ hours of receipt, by e-mail to: analyst@rsc.org 


\section{Queries for the attention of the authors}

Journal: Analyst

Paper: c5an00525f

Title: Dielectric spectroscopy for monitoring human pancreatic islet differentiation within cell-seeded scaffolds in a perfusion bioreactor system

Editor's queries are marked like this [Q1, Q2,...], and for your convenience line numbers are indicated like this $[5,10,15, \ldots]$.

Please ensure that all queries are answered when returning your proof corrections so that publication of your article is not delayed.

\begin{tabular}{|l|l|l|}
\hline $\begin{array}{l}\text { Query } \\
\text { Reference }\end{array}$ & Query & Remarks \\
\hline Q1 & $\begin{array}{l}\text { For your information: You can cite this article before you } \\
\text { receive notification of the page numbers by using the following } \\
\text { format: (authors), Analyst, (year), DOI: 10.1039/c5an00525f. }\end{array}$ & $\begin{array}{l}\text { Please carefully check the spelling of all author names. This is } \\
\text { important for the correct indexing and future citation of your } \\
\text { article. No late corrections can be made. }\end{array}$ \\
\hline Q2 & Ref. 15, 39 and 57: Please provide the page (or article) number(s). & \\
\hline Q3 & Ref. 50: Please provide the year of publication. & \\
\hline Q4 & &
\end{tabular}




\title{
Dielectric spectroscopy for monitoring human pancreatic islet differentiation within cell-seeded scaffolds in a perfusion bioreactor system
}

\author{
J. Daoud, ${ }^{a}$ K. Heileman, ${ }^{a}$ S. Shapka, ${ }^{a}$ L. Rosenberg ${ }^{b}$ and M. Tabrizian ${ }^{\star a, c}$
}

The long-term in vitro culture and differentiation of human pancreatic islets is still hindered by the inability to emulate a suitable microenvironment mimicking physiological extracellular matrix (ECM) support and nutrient/oxygen perfusion. This is further amplified by the current lack of a non-invasive and rapid monitoring system to readily evaluate cellular processes. In this study, we realized a viable method for noninvasively monitoring isolated human pancreatic islets in vitro. Islets are induced to dedifferentiate into proliferative duct-like structures (DLS) in preparation for potential and subsequent re-differentiation into functional islet-like structures (ILS) in a process reminiscent of islet regeneration strategies. This longterm in vitro process is conducted within a three-dimensional microenvironment involving islets embedded in an optimized ECM gel supported by microfabricated three-dimensional scaffolds. The isletscaffold is then housed and continuously perfused within chambers of a bioreactor platform. The process in its entirety is monitored through dielectric spectroscopy measurements, yielding an accurate representation of cellular morphology, functionality, and volume fraction. This non-invasive and real-time monitoring tool can be further manipulated to elucidate important information about the optimized cellular microenvironment required for maintaining long-term culture and achieve efficient differentiation for islet regeneration.

Received 16th March 2015 Accepted 29th July 2015

DOI: 10.1039/c5an00525f

www.rsc.org/analyst re-differentiation into functionally viable ILS, thus arriving at an islet mass sufficient for multiple transplantations. In order to accomplish this goal, it is important to employ a scaffold microenvironment for islet culture coupled to a perfusion type system to ensure high mass and nutrient transfer characteristics. In addition, a non-invasive monitoring system should be incorporated in order to provide real-time measurements indicative of the differentiation progress and cellular processes occurring within the islet culture bioreactor. In this study, we hypothesize that pancreatic islets, embedded in a developed ECM-derived gel within geometrically controlled scaffolds, may be induced to differentiate within a designed bioreactor perfusion system whilst incorporating parallel plate platinum electrodes allowing real-time dielectric spectroscopy monitoring.

Adequate functioning of implantable human pancreatic islets depends upon the re-establishment of the cell-matrix interactions, reduction of islet immunogenicity, and facilitation of islet implantation to further improve allograft survival. $^{2}$ It is therefore imperative to arrive at a three-dimensional microenvironment, within a tissue bioreactor setting, in order to emulate physiological conditions. We have successfully demonstrated in past studies the importance of re-establishing the basement membrane ECM components for islet survival

\footnotetext{
${ }^{a}$ Biomedical Engineering Department, McGill University, 3775 University Street, Montreal, Quebec H3A 2B4, Canada.E-mail: maryam.tabrizian@mcgill.ca; Fax: +1-514-398-7461; Tel: +1-514-398-8129

${ }^{b}$ Department of Surgery, Faculty of Medicine, McGill University, 3655 Promenade Sir William Osler, Montreal, Quebec, H3G 1 Y6 Canada

${ }^{c}$ Faculty of Dentistry, McGill University, 3775 University Street, Montreal, Quebec H3A 2B4, Canada
}

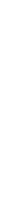

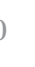

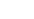

年

40

s.


in vitro. ${ }^{3}$ Moreover, we introduced a geometrically controlled polymer scaffold system incorporating embedded human pancreatic islets within an optimized ECM gel microenvironment, thus allowing for long-term culture maintaining the cellular functional viability. ${ }^{4}$

In addition to arriving at a suitable islet microenvironment, it is also critical to develop a reactor system in which islets may be monitored non-invasively to observe cellular processes of proliferation, differentiation, as well as viability. Recently, rotary cell culture system (RCCS) bioreactors, ${ }^{5}$ which simulate microgravity, have been developed. Simulated microgravity involves rotating cultured cells by suspending them in bioreactors such as the high aspect ratio vessel (HARV). Consequently, cells suspended in the HARV undergo continuous free-fall at terminal velocity with low hydrodynamic shear stress, low turbulence, and high mass transfer of nutrients. ${ }^{6-9}$ However, a major drawback of such a system is its inability to monitor the islet processes without the need for stopping the culture process, obtaining samples, and performing invasive, long and arduous procedures to monitor cellular processes. Additionally, such systems lack a three-dimensional support for the cultured islets, which are needed to support and protect cells and to provide three-dimensional culture conditions in vitro. ${ }^{10-13}$ To address the lack of islet monitoring systems, several studies have been performed in order to adapt real-time biosensing of islets in vitro using primitive microfluidic devices; however they lack the important coupling to a viable reactor system, as well as a cost-effective, high throughput method that provides information concerning the morphological and proliferative state of the islets. ${ }^{14-19}$ Recently, we have demonstrated the viability of employing a real-time, noninvasive monitoring system based on dielectric spectroscopy which allows for the measurement of epithelial cell proliferation into cellular clusters and subsequent differentiation into cyst-like structures - analogous to the desired process of pancreatic islet differentiation into DLS. ${ }^{20}$

Dielectric responses of biological cells to radio-frequency AC fields are mainly due to interfacial polarization in the heterogeneous structure of cells. ${ }^{21-24}$ Since the surface and internal cell membranes are considerably less conductive than the cytoplasm and the external medium, the membrane topology plays an important role in interfacial polarization. The membranes are polarized depending on the frequency of the applied AC field; therefore, a cell suspension shows dielectric dispersion, i.e., frequency dependence of complex permittivity $\varepsilon^{*}$ defined as

$$
\varepsilon^{*}=\varepsilon+\frac{\kappa}{\mathrm{j} \omega \varepsilon_{0}}
$$

where $\varepsilon$ is relative permittivity, $\kappa$ electric conductivity, $\varepsilon_{0}$ the permittivity of a vacuum, $\mathrm{j}^{2}=-1, \omega$ angular frequency defined by $\omega=2 \pi f$ and $f$ the frequency of the applied AC field. Analysis of the dielectric spectrum requires modeling of cells. Derived dielectric parameters may be related to cellular functionality in response to stimuli ${ }^{25}$ as well as variations in electrical properties. ${ }^{26}$ In addition, dielectric parameters can further be correlated to cellular differentiation through variations arising from morphological changes. ${ }^{20,27}$ For simple cells modeled as spherical and ellipsoidal shell models, we can use analytical equations that relate the electric parameters of the cell components to the dielectric spectrum; this is accomplished using previously derived equations based on Maxwell-Wagner effects such as the single/double shell, vesicle inclusion and composite cell models. ${ }^{21,28,29}$ However, intricate cellular structures such as cell cluster spheroids and islets possess complex structures, for which analytical equations may be limited. Therefore numerical simulation techniques such as finite element analysis (FEA) are employed to better characterize the effects of irregular shapes as well as gap/tight junctions connecting cell aggregates. ${ }^{28,30-34}$ They may then be related to appropriate analytical cell models, employing appropriate thin layer approximations (TLA), ${ }^{35}$ to better characterize membrane and cytoplasmic permittivity/conductivity.

We therefore present an effective strategy for a human islet in vitro culture system which incorporates the aforementioned requirements: (i) an ECM microenvironment within a three-dimensional geometrically controlled, microfabricated scaffold; (ii) a bioreactor platform that allows for continuous and controlled media perfusion and nutrient/oxygen transport; and (iii) the incorporation of a real-time, non-invasive cellular monitoring system based on dielectric spectroscopy through changes in dielectric parameters measured across multiple culture chambers. This makes possible the realization of the goal of islet long-term culture and differentiation in vitro, based on the dedifferentiation of islets to proliferative DLS, thus allowing for their potential and subsequent re-differentiation into functional ILS, ${ }^{36,37}$ whilst being monitored using a dielectric measurement system.

\section{Materials and methods}

\section{Human islet isolation}

Pancreata from adult human cadaveric organ donors were obtained through the local organ procurement organization, Quebec Transplant, and were isolated according to established protocols $^{38}$ reviewed by the McGill Ethical Committee. Briefly, following removal of the organ, cold ischemia time was no more than $8 \mathrm{~h}$ prior to islet isolation. The main pancreatic duct was cannulated and perfused with Liberase HI (Roche Diagnostics, Montreal, QC). The perfused organ was placed in a closed system (Ricordi Apparatus) and heated to $37^{\circ} \mathrm{C}$ to activate the enzyme blend. Following the appearance of free islets in samples, the system was cooled and free tissues were collected and washed. Tissues were applied to a continuous density gradient created using Ficoll (Biochrom KG, Berlin, Germany) in a cell processor (COBE). Free islets with diameters ranging from 75 to $400 \mu \mathrm{m}$, determined to be greater than $80 \%$ pure by staining with dithizone (Sigma, St Louis, MO), a zinc chelator, were collected and washed. IHC to detect the presence of amylase and cytokeratin was negative, consistent with the absence of ductal and exocrine tissue. 


\section{Modelling the phases of islet differentiation}

Islet differentiation occurs through a two-step mechanism, which is modeled using the Computer Aided Design (CAD) model in Fig. 1A. This model employed in the Finite Element Analysis (FEA) simulation is composed of three overlapping layers of dodecahedra, possessing 12 faces of 6 sides each $\sim 10 \mu \mathrm{m}$ in length, representing cells. The first layer is the central cell; the second layer is composed of 12 cells adjoined to each face of the first layer dodecahedron cell; the third layer represents the 32 outer cells again adjoined to each face of the second layer dodecahedron cells. This gives a total of 45 cells representing an islet cellular cluster (phase 'I'), while the DLS is represented by only the third outer layer of 32 cells giving the desired cellular cyst structure (phase 'II'). Both phases measure $56 \mu \mathrm{m}$ in diameter. Fig. 1B shows a cross-section of adjoined dodecahedron faces, whereby adjacent cells are interconnected through $100 \mathrm{~nm}$ wide gap junctions and a tight junction barrier separated by $100 \mathrm{~nm}$ representing fenestration gaps between cells. Schematic representations of pancreatic islet clusters and differentiated DLS are shown in Fig. 1C and $\mathrm{D}$, respectively. In addition, the associated numerical cell models employed to represent islets (Vesicle Inclusion; VI model) and DLS (Composite Cell; CC model) are given in

\section{Fig. 1E and F, respectively.

A)
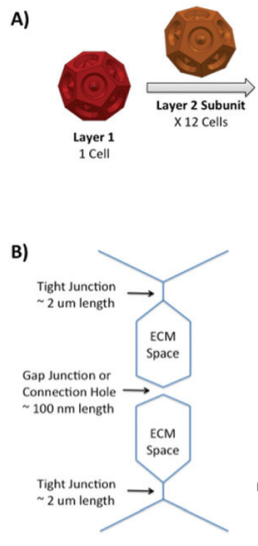

F)
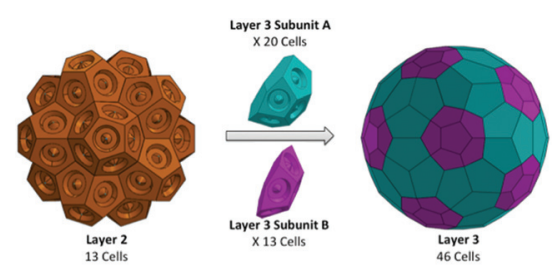

c)

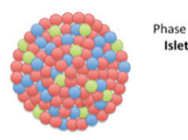

D)
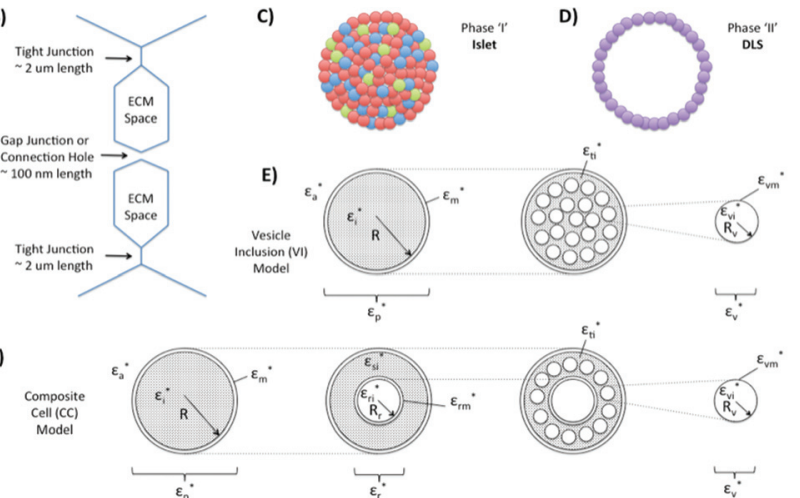

Fig. 1 (A) Schematic of the configuration of an islet for FEA comprising 3 layers, composed of dodecahedron geometries representing cells. First, a central cell containing 12 faces is joined to a second layer of 12 cells, followed by 33 cells at the third layer periphery, giving a total of 46 cells. (B) A cross-section of a dodecahedron adjoining faces sandwiching ECM space, showing the $\sim 100 \mathrm{~nm}$ gap junction, as well as 2 sets of $2 \mu \mathrm{m}$ tight junctions, with faces separated by $100 \mathrm{~nm}$ fenestrations. To perform the FEA, the process of islet differentiation is described by (C) phase ' $I$ ' and (D) phase 'II' depicting the islet and DLS, respectively. The analytical cell models of (E) the vesicle inclusion ( $\mathrm{VI}$ ) and (F) the composite cell (CC) and the respective dielectric parameters are used to describe the islet and DLS phases, respectively.

\section{Numerical calculation of complex permittivity by FEA}

1

Numerical calculations were performed by FEA using COMSOL Multiphysics with an AC/DC module (COMSOL, Sweden). Quasi-electrostatic and time harmonics conditions were adopted over a frequency range from $1 \mathrm{kHz}$ to $100 \mathrm{MHz}$. To reduce computational tasks, we adopted the 'thin-layer' approximation that deals with the membrane as an interface of zero thickness, as described in a previous paper. ${ }^{39}$ We consider subdomains $i$ and a, corresponding to the cytoplasm and the external medium, which have complex conductivities of $\kappa_{\mathrm{i}}^{*}$ and $\kappa_{\mathrm{a}}^{*}$, respectively. Complex conductivity $\kappa^{*}$ is simply related to complex permittivity $\varepsilon^{*}$ as $\kappa^{*}=\mathrm{j} \omega \varepsilon_{0} \varepsilon^{*}$. The interface between the subdomains has the impedance of $d / \kappa_{\mathrm{m}}^{*}$, where $d$ is the membrane thickness and $\kappa_{\mathrm{m}}^{*}$ is the complex conductivity of the membrane. The potential distribution was solved under the following boundary conditions. For the four sides of the cube, the current density is zero, i.e., electric insulation conditions. The top and the bottom of the cube have potentials of $1 \mathrm{~V}$ and $0 \mathrm{~V}$, respectively. The current density $J_{\mathrm{m}}$ through the interface between subdomains $i$ and $a$ is given by

$$
\begin{gathered}
-n_{\mathrm{i}} \cdot k_{\mathrm{i}}^{*} \nabla V_{\mathrm{i}}=n_{\mathrm{a}} \cdot k_{\mathrm{a}}^{*} \nabla V_{\mathrm{a}}=J_{\mathrm{m}} \\
J_{\mathrm{m}}=\frac{\kappa_{\mathrm{m}}^{*}}{d}\left(V_{\mathrm{i}}-V_{\mathrm{a}}\right)
\end{gathered}
$$

where $n_{\mathrm{i}}$ and $n_{\mathrm{a}}$ are the outward normals to the boundaries of subdomains $\mathrm{i}$ and a, and $V_{\mathrm{i}}$ and $V_{\mathrm{a}}$ are the electric potentials of subdomains $i$ and a at the interface.

The current through the cube is calculated by integrating the current density over the top or the bottom boundary of the cube. The current and the applied voltage provide the admittance $Y$ of the cubic system of $l \times l \times l$. The admittance is simply converted to the effective complex conductivity $\kappa^{*}$ and complex permittivity $\varepsilon^{*}$ as

$$
\kappa^{*}=\mathrm{j} \omega \varepsilon_{0} \varepsilon^{*}=\frac{Y}{l}=\kappa+\mathrm{j} \omega \varepsilon_{0} \varepsilon
$$

\section{Scaffold fabrication}

Scaffolds were fabricated according to a previously described protocol. ${ }^{4,20}$ Briefly, the plotting material was prepared by dissolving poly(DL-lactide-co-glycolide) acid (PLGA; $85 \mathrm{~mol} \%$ DL-lactide, $15 \mathrm{~mol} \%$ glycolide; molecular weight of 91.64-141.52 kDa) (Lakeshore Biomaterials, Birmingham, AL) in methyl ethyl ketone (MEK) (Sigma). Subsequently, the solution was mixed with $15 \% \mathrm{w} / \mathrm{w}$ of sodium chloride $(\mathrm{NaCl})$ particles. The $\mathrm{NaCl}$ porogen was previously sieved to obtain a desired particle size distribution $(53-63 \mu \mathrm{m})$. The optimal concentration of the polymer in the solvent was determined based on the viscosity constraints of the 3D plotter while targeting optimal syringe deposition. It was found that a $1: 1$ ratio of PLGA to solvent provided an adequate viscosity without compromising the smooth deposition of the paste after incorporation of the porogen.

Three-dimensional scaffolds were fabricated using an XYZ bioplotter (Envisiontec, Gladbeck, Germany), previously 
described by Landers et al. ${ }^{40}$ The PLGA polymer solution was transferred to the plotting cartridge and was dispensed layer by layer, forming a $0^{\circ} / 90^{\circ}$ strand structure using a CAD file as a template. The fabricated bricks were $40 \times 40 \mathrm{~mm}$-wide and $3.6 \mathrm{~mm}$-thick. Scaffold was punched into $8 \mathrm{~mm}$-diameter, $3.6 \mathrm{~mm}$-thick discs, and then immersed in water at $50{ }^{\circ} \mathrm{C}$ for up to $6 \mathrm{~h}$ to extract the salt particles, air-dried for $24 \mathrm{~h}$, and vacuum-dried for $48 \mathrm{~h}$ to allow complete evaporation of the solvent/water.

Dispensing tips of $250 \mu \mathrm{m}$ diameter were used to yield a strand diameter of $\sim 150 \mu \mathrm{m}$ at a dispensing speed of $165 \mathrm{~mm}$ $\mathrm{s}^{-1}$. ${ }^{41}$ The layers were overlaid to give a strand thickness of $120 \mu \mathrm{m}$. Fabricated scaffolds possessed a pore size of $400 \mu \mathrm{m}$. The optimal distance between the strands was determined by targeting a high scaffold effective surface available for cell attachment, while providing full interconnectivity and a sufficient pore size for pancreatic islet seeding. The effective surface of the 3D plotted constructs was determined as a function of scaffold plotting parameters.

\section{Scaffold preparation and priming}

Scaffolds with $400 \mu \mathrm{m}$ pore size, diameter of $8 \mathrm{~mm}$ and thickness of $3.6 \mathrm{~mm}$ were exposed to UV light under a laminar tissue culture hood for $20 \mathrm{~min}$. The scaffolds were then immersed in $70 \%$ ethanol for $5 \mathrm{~min}$, and then washed and suspended with serum-free CMRL media (Invitrogen, Grand Island, NY) for $2 \mathrm{~h}$ at $37{ }^{\circ} \mathrm{C}$ for priming. Scaffolds were then dried with sterile blotting paper under a laminar tissue culture hood to prepare for islet seeding.

\section{Reactor design and fabrication}

The design of the tissue bioreactor followed perfusion-based modeling. Poly(methyl methacrylate) (PMMA) was purchased from McMaster-Carr (Hamilton, Ontario), and the fabrication process took place in the McGill Biomedical Engineering machine shop. Machining was performed using a knee-type Topwell 3VK turret milling machine (Topwell Co., Taiwan) that has been retrofitted with a Fagor 8040MC (Fagor Automation, Korea) computer numerical controller (CNC). The bioreactor dimensions were $100 \times 40 \mathrm{~mm}$, composed of a PMMA base, center, and top. PMMA thermoplastic was chosen due to its biocompatibility, ease of machining, and transparent nature that serves to better visualize the sample and media. The base and top components were $3 \mathrm{~mm}$ thick and housed 4 copper leads ( $1 \mathrm{~mm}$ thick; $5 \times 20 \mathrm{~mm}$ ) and $20 \mathrm{~mm}$-diameter, $1 \mathrm{~mm}$ thick platinum electrodes that were aligned at each end. Grooves, $0.5 \mathrm{~mm}$ in depth, were machined on PMMA surfaces outlining the position of copper leads and platinum electrodes in order to facilitate alignment and subsequent bonding. Loctite all-purpose epoxy glue (Henkel AG \& Company, Germany) was used to permanently bind metal components to plastic and avoid delamination. The center was $6 \mathrm{~mm}$ thick and housed 4 multiple chambers $5 \mathrm{~mm}$ in diameter for islet culture within the post-processed scaffolds. The 4 multiple chambers allow for parallel replicate experiments to be performed. Each chamber possessed a unique inlet/outlet that
A)

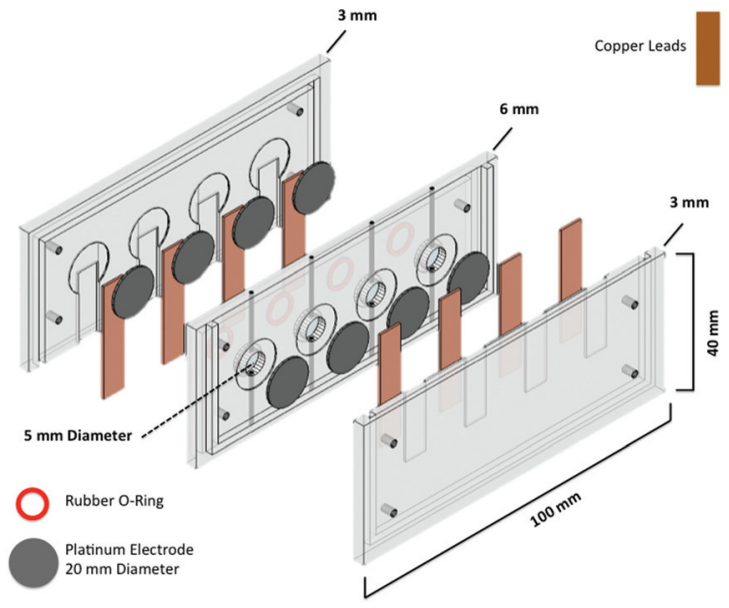

1

B)

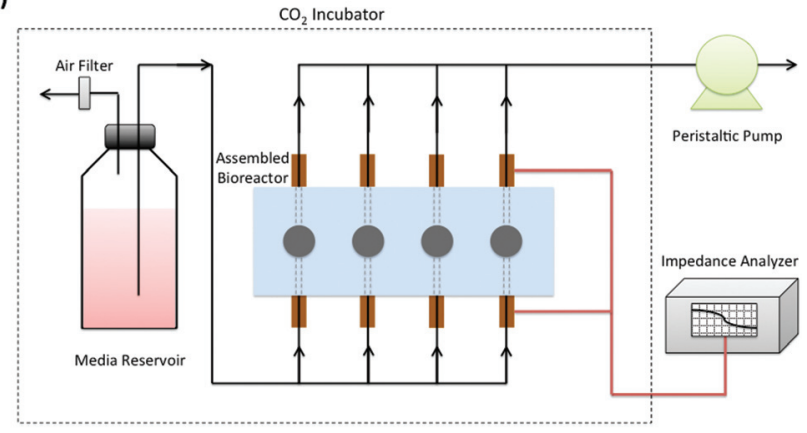

Fig. 2 Design schematic of the electrode-equipped bioreactor system employed in this study for dielectric spectroscopy monitoring of scaffold-based islet differentiation. (A) Outline of the components and arrangement comprising the four chambered perfusion-based bioreactor system showing the culture chambers sandwiched between platinum measurement electrodes with the respective copper leads. (B) Diagram of the perfusion bioreactor system set-up with media reservoir enclosed within a $\mathrm{CO}_{2}$ incubator, as well as peristaltic pump and impedance analyzer configuration.

was perfused via $1 \mathrm{~mm}$-diameter channels. Individual perfusion and outlet channels were selected in order to avoid cross-contamination between samples. A schematic of the reactor is shown in Fig. 2A. The electrodes were carefully plated with platinum black to reduce the parasitic capacitance due to the electrode polarization. ${ }^{42-44}$

\section{Islet cell seeding and culture}

Human islets were suspended in an optimized ECM milieu that is detailed in an earlier paper. ${ }^{4}$ Briefly, islets were suspended in $4 \mathrm{mg} \mathrm{ml}^{-1}$ type I rat tail collagen supplemented with $100 \mu \mathrm{g} \mathrm{ml} \mathrm{m}^{-1}$ of fibronectin and collagen IV (BD Biosciences, San Jose, CA). The suspension was then neutralized with a $3: 2$ ratio of $10 \times$ Waymouth media (Sigma Aldrich, Oakville, Ontario) and $0.34 \mathrm{~N} \mathrm{NaOH}$ (known as neutralization solution), respectively, to induce gelling. The seeding technique used is known as cell-gel seeding, ${ }^{45}$ which provides a uniform cell distribution. Scaffolds were placed in 48-well pates, and then seeded through the immersion with $350 \mu \mathrm{L}$ of the neutralized gel suspension containing 500 IEQ (Islet Equivalents). 
Following neutralization, the islets were transferred to different wells of the reactor, leaving one well for a scaffold seeded with an ECM mixture without islets, serving as a negative control. The reactor was placed inside a small $\mathrm{CO}_{2}$ incubator and perfused continuously with serum-free DMEM/F12, containing Cholera Toxin (CT) (Sigma Aldrich, Oakville, Ontario), at a flow rate of $50 \mu \mathrm{min}^{-1}$. This process was continued for 9 days until the islets have completely transformed into DLS structures. In order to confirm that de-differentiation was occurring, and to supplement the dielectric data, a scaffold was sacrificed every day in order to obtain microscopic images. At day 9, the complete transformation into DLS should be accomplished. The set-up of the continuous culture experiment is shown in Fig. 2B.

\section{Dielectric measurements}

At 24 hour intervals, dielectric spectra for capacitance and conductance were carried out using an Agilent 4294a impedance analyzer (Palo Alto, CA) at $300 \mathrm{mV}$ and the collected data were converted to permittivity and conductivity values. Throughout the islet differentiation process, the measured values were analyzed and fitted to analytical Effective Media Approximations (EMAs), as well mathematical cellular models derived from frequency dependent permittivity dispersions described by Maxwell-Wagner effects. These mathematical models depict the cellular morphology and volume fraction in order to arrive at relations accurately describing cellular activity within the measured scaffolds.

\section{Curve fitting and data analysis}

The measured dielectric spectra were characterized using the Cole-Cole relaxation equation:

$$
\varepsilon^{*}=\varepsilon_{\mathrm{h}}+\sum_{i=1}^{n} \frac{\Delta \varepsilon_{n}}{1+(\mathrm{j} \omega \tau)^{\beta_{n}}}+\frac{\kappa_{1}}{\mathrm{j} \omega \varepsilon_{0}}
$$

where $n$ is the number of sub-dispersions, $\varepsilon_{\mathrm{h}}$ the highfrequency limit of relative permittivity, $\Delta \varepsilon$ the relaxation intensity, $\tau$ the relaxation time $\left(\tau=1 /\left(2 \pi f_{\mathrm{c}}\right)\right.$, where $f_{\mathrm{c}}$ is the characteristic frequency), $\kappa_{1}$ the low-frequency limit of conductivity, and $\beta$ is the Cole-Cole parameter $(0<\beta \leq 1)$. The relaxation parameters $\Delta \varepsilon, \tau$ or $f_{\mathrm{c}}, \varepsilon_{\mathrm{h}}$ and $\kappa_{\mathrm{l}}$ were obtained by fitting eqn (5) to the dielectric spectra. The electrode polarization effect was corrected using the function described in a previous paper. ${ }^{46}$ The curve-fitting was performed by the non-linear least-squares optimization with the Levenberg-Marquardt algorithm, implemented in MATLAB software (Natick, MA). After correction for the electrode polarization effect, the dielectric spectra were analyzed using theoretical models based on interfacial polarization (or the Maxwell-Wagner polarization). According to the phases of islet differentiation, VI and CC models (Fig. 1E and F) were adopted in order to elucidate cellular characteristics such as cell membrane capacitance.

We hypothesize that the islet resembles a spherical shell structure in which the intershell space is populated by spherical aggregates; the islet outer shell is composed of inter- connected membranes of cells on the periphery that are in contact with the extracellular matrix. The VI model as shown in Fig. $1 \mathrm{E}$ is therefore employed, where the islet is regarded as a shell enclosing many inner spherical inclusions or cell aggregates that are connected with tight junctions. The equivalent homogeneous complex permittivity of the islet cell aggregate $\varepsilon_{\mathrm{p}}^{*}$ is given by the Maxwell-Wagner relation:

$$
\varepsilon_{\mathrm{p}}^{*}=\varepsilon_{\mathrm{m}}^{*} \frac{2(1-v) \varepsilon_{\mathrm{m}}^{*}+(1+2 v) \varepsilon_{\mathrm{i}}^{*}}{(2+v) \varepsilon_{\mathrm{m}}^{*}+(1-v) \varepsilon_{\mathrm{i}}^{*}}
$$

where subscripts $\mathrm{m}$ and $\mathrm{i}$ of $\varepsilon^{*}$ denote the membrane and the interior, while $v=\left[R /\left(R+d_{\mathrm{m}}\right)\right]^{3}$ in which $R$ is the islet aggregate radius and $d_{\mathrm{m}}$ is the thickness of the outer shell, or the equivalent of the membrane thickness of the islet peripheral cells. The complex permittivity of the aggregate interior, $\varepsilon_{i}^{*}$, is composed of a mixture of inner spheres described by the HanaiAsami-Koizumi EMA:

$$
\frac{\varepsilon_{\mathrm{i}}^{*}-\varepsilon_{\mathrm{v}}^{*}}{\varepsilon_{\mathrm{ti}}^{*}-\varepsilon_{\mathrm{v}}^{*}}\left(\frac{\varepsilon_{\mathrm{ti}}^{*}}{\varepsilon_{\mathrm{i}}^{*}}\right)^{1 / 3}=1-P_{\mathrm{i}}
$$

where $P_{\mathrm{i}}$ is the volume fraction of cells within the aggregate and subscript ti denotes the intershell space between cells. It is imperative to use HAK EMA, since it best describes a mixture equation that incorporates far-field interactions between spherical particles; thus it is most appropriate to be applied to high volume fraction suspensions. The complex permittivity of the inner cells, $\varepsilon_{\mathrm{v}}^{*}$, is given by:

$$
\varepsilon_{\mathrm{v}}^{*}=\varepsilon_{\mathrm{vm}}^{*} \frac{2\left(1-v_{2}\right) \varepsilon_{\mathrm{vm}}^{*}+\left(1+2 v_{2}\right) \varepsilon_{\mathrm{vi}}^{*}}{\left(2+v_{2}\right) \varepsilon_{\mathrm{vm}}^{*}+\left(1-v_{2}\right) \varepsilon_{\mathrm{vi}}^{*}}
$$

where subscripts vm and vi denote the membrane and the cytoplasm of the individual cells within the islet aggregate, while $v_{2}=\left[R_{\mathrm{v}} /\left(R_{\mathrm{v}}+d_{\mathrm{vm}}\right)\right]^{3}$ in which $R_{\mathrm{v}}$ and $d_{\mathrm{vm}}$ represent the radius and membrane thickness of the inner cells, respectively.

We further hypothesize that the DLS structure is composed of an outer shell and an inner concentric sphere representing the lumen. The CC model as shown in Fig. $1 \mathrm{~F}$ is therefore employed, where the intershell space contains a single layer of epithelial cell aggregates connected through tight junctions, thus forming a cystic structure. The interconnected cell membranes thus form both the outer and inner shells, bordering both the periphery and the lumen. As in the VI model, the cystic DLS is described by the complex permittivity in eqn (6). However, in the CC model (Fig. 1F), the complex permittivity of the interior, $\varepsilon_{\mathrm{i}}^{*}$, is composed of an inner concentric sphere $\left(\varepsilon_{\mathrm{r}}^{*}\right)$ and cell aggregate layer of the intershell space $\left(\varepsilon_{\mathrm{si}}^{*}\right)$ :

$$
\varepsilon_{\mathrm{i}}^{*}=\varepsilon_{\mathrm{si}}^{*} \frac{2\left(1-v_{2}\right) \varepsilon_{\mathrm{si}}^{*}+\left(1+2 v_{2}\right) \varepsilon_{\mathrm{r}}^{*}}{\left(2+v_{2}\right) \varepsilon_{\mathrm{si}}^{*}+\left(1-v_{2}\right) \varepsilon_{\mathrm{r}}^{*}}
$$

with the equivalent complex permittivity $\varepsilon_{\mathrm{r}}^{*}$ of the inner membrane-covered core sphere, or lumen, given by:

$$
\varepsilon_{\mathrm{r}}^{*}=\varepsilon_{\mathrm{rm}}^{*} \frac{2\left(1-v_{3}\right) \varepsilon_{\mathrm{rm}}^{*}+\left(1+2 v_{3}\right) \varepsilon_{\mathrm{ri}}^{*}}{\left(2+v_{3}\right) \varepsilon_{\mathrm{rm}}^{*}+\left(1-v_{3}\right) \varepsilon_{\mathrm{ri}}^{*}}
$$


where subscripts of si, rm and ri of $\varepsilon^{*}$ refer to the intershell region between the outer and inner spheres, the inner shell and the inner sphere space or lumen, respectively, while $v_{2}=$ $\left[\left(R_{\mathrm{r}}+d_{\mathrm{rm}}\right) / R\right]^{3}$ and $v_{3}=\left[R_{\mathrm{r}} /\left(R_{\mathrm{r}}+d_{\mathrm{rm}}\right)\right]^{3}$ in which $R_{\mathrm{r}}$ and $d_{\mathrm{rm}}$ represent the radius and thickness of the inner shell, containing the lumen. Since the intershell space is composed of a densely packed interconnected cell layer, the HAK EMA is once again utilized to yield $\varepsilon_{\mathrm{si}}^{*}$ :

$$
\frac{\varepsilon_{\mathrm{si}}^{*}-\varepsilon_{\mathrm{v}}^{*}}{\varepsilon_{\mathrm{ti}}^{*}-\varepsilon_{\mathrm{v}}^{*}}\left(\frac{\varepsilon_{\mathrm{ti}}^{*}}{\varepsilon_{\mathrm{si}}^{*}}\right)^{1 / 3}=1-P_{\mathrm{i}}
$$

where $P_{\mathrm{i}}$ is the volume fraction of cells within the cyst and subscript ti denotes the intershell space between cells. The complex permittivity of the inner cells, $\varepsilon_{\mathrm{v}}^{*}$, is given by:

$$
\varepsilon_{\mathrm{v}}^{*}=\varepsilon_{\mathrm{vm}}^{*} \frac{2\left(1-v_{4}\right) \varepsilon_{\mathrm{vm}}^{*}+\left(1+2 v_{4}\right) \varepsilon_{\mathrm{vi}}^{*}}{\left(2+v_{4}\right) \varepsilon_{\mathrm{vm}}^{*}+\left(1-v_{4}\right) \varepsilon_{\mathrm{vi}}^{*}}
$$

where subscripts vm and vi denote the membrane and cytoplasm of the individual cells within the intershell space, while $v_{4}=\left[R_{\mathrm{v}} /\left(R_{\mathrm{v}}+d_{\mathrm{vm}}\right)\right]^{3}$ in which $R_{\mathrm{v}}$ and $d_{\mathrm{vm}}$ represent the radius and membrane thickness of the cells, respectively.

In the case of islet and DLS clusters - described by VI and CC models, respectively - the outer shell $\left(\varepsilon_{\mathrm{m}}^{*}\right)$ represents the basement membrane matrix, composed of ECM proteins, at a thickness of $50 \mathrm{~nm},{ }^{47,48}$ while assuming the same dielectric parameters as the surrounding media $\left(\varepsilon_{\mathrm{a}}^{*}\right)$, intershell space $\left(\varepsilon_{\mathrm{ti}}^{*}\right)$, and cell cytoplasm $\left(\varepsilon_{\mathrm{vi}}^{*}\right)$. The inner shell $\left(\varepsilon_{\mathrm{rm}}^{*}\right)$ in the CC model of DLS also assumes basement membrane characteristics equal to the $\varepsilon_{\mathrm{m}}^{*}$ outer shell - applying as well the same parameters to the inner sphere lumen $\left(\varepsilon_{\mathrm{ri}}^{*}\right)$. These equivalence relations can be summed up as:

$$
\varepsilon_{\mathrm{m}, \mathrm{rm}}^{*}=\varepsilon_{\mathrm{a}, \mathrm{ti}, \mathrm{vi}, \mathrm{ri}}^{*}
$$

When the VI and CC models are suspended in the medium of $\varepsilon_{\mathrm{a}}^{*}$ at a low volume fraction $P$, the complex permittivity of the suspension $\varepsilon^{*}$ becomes

$$
\varepsilon^{*}=\varepsilon_{\mathrm{a}}^{*} \frac{2(1-P) \varepsilon_{\mathrm{a}}^{*}+(1+2 P) \varepsilon_{\mathrm{p}}^{*}}{(2+P) \varepsilon_{\mathrm{a}}^{*}+(1-P) \varepsilon_{\mathrm{p}}^{*}}
$$

which is defined as the Pauly-Schwan (PS) equation (Pauly and Schwan, 1959), at overall volume fraction $P$.

\section{Immunofluorescence}

The morphology of the human islets was observed using phase contrast microscopy and confocal microscopy. Samples were prepared as previously described..$^{49}$ Briefly, samples were fixed in $4 \%$ formalin for $30 \mathrm{~min}$ at room temperature. Fixed structures were washed with PBS-glycine $(130 \mathrm{~mm} \mathrm{NaCl}, 7 \mathrm{~mm}$ $\mathrm{Na}_{2} \mathrm{HPO}_{4}, 100 \mathrm{~mm}$ glycine) three times for $10 \mathrm{~min}$ each. The structures were then blocked in IF buffer $(130 \mathrm{~mm} \mathrm{NaCl}, 7 \mathrm{~mm}$ $\mathrm{Na}_{2} \mathrm{HPO}_{4}, 3.5 \mathrm{~mm} \mathrm{NaH} \mathrm{PO}_{4}, 7.7 \mathrm{~mm} \mathrm{NaN}, 0.1 \%$ bovine serum albumin, $0.2 \%$ Triton X-100, $0.05 \%$ Tween 20 ) plus $10 \%$ goat serum (Invitrogen) for $1 \mathrm{~h}$ at room temperature. This was followed by a secondary block with IF buffer containing $10 \%$ goat serum and $20 \mu \mathrm{g}$ of goat anti-mouse $\mathrm{F}\left(\mathrm{ab}^{\prime}\right)_{2}$ (Jackson Immunochemicals, West Grove, PA) for $1 \mathrm{~h}$. Primary antibodies were diluted in secondary blocking buffer, followed by incubation overnight at $4{ }^{\circ} \mathrm{C}$. The primary antibodies used were guinea pig anti-insulin $(1: 100)$ and rabbit anti-CK19 $(1: 500)$. Samples were washed three times in IF buffer for 20 min each. Cy3-conjugated anti-guinea pig and FITC-conjugated anti-rabbit secondary antibodies (Abcam, Cambridge, MA) were diluted (1:500) in IF buffer containing 10\% goat serum, followed by incubation for $1 \mathrm{~h}$. After washing three times with IF buffer (20 min each), structures were incubated with $0.5 \mathrm{ng} \mathrm{ml}^{-1}$ of Hoechst 33342 (Molecular Probes). A final rinse with IF for $5 \mathrm{~min}$ at room temperature was followed by mounting with the anti-fade prolong (Molecular Probes) and allowed to dry at room temperature. Confocal analysis was performed by using the Zeiss LSM 510 confocal system. Images were generated by using Zeiss AxioVision software.

\section{Statistical analysis}

The number of biological replicates is $n=5$ for all experiments performed. Statistical significance was determined by one-way ANOVA with a post hoc Bonferroni's test or a paired Student's $t$ test, where applicable. Differences were considered significant when $P<0.05$ or less. Data fitting to the Cole-Cole relation in eqn (5) possessed an $R^{2}$ value of $>0.99$, while fitting of dielectric spectra to analytical cell models produced an $R^{2}$ value of $>0.95$.

\section{Results and discussion}

\section{FEA simulation of islet differentiation}

Prior to performing characterization of islet differentiation by dielectric spectroscopy, it is important to examine the theoretical relaxation curves calculated from models at the differentiation phases in Fig. 1C-F in order to determine whether the phases exhibit markedly different dielectric responses that are easily distinguishable. The model of phase 'I' corresponds to the pancreatic islet cell cluster, whereas the model of phase ' $\mathrm{II}$ ' represents the DLS cystic structure, with adjacent cells connected to each other with gap/tight junctions, possessing a spherical lumen at the center enclosed by connected cells. The porosity of cell clusters within the islet and DLS was assumed to be $5 \%$, in the range simulated in the literature for diffusion kinetics within islets. ${ }^{50}$ Assigning VI and CC models for islets (phase ' $\mathrm{I}$ ') and DLS (phase 'II'), respectively, FEA calculations were carried out with the following parameter values: $P=$ $0.0585, P_{\mathrm{i}}=0.95 \varepsilon_{\mathrm{m}}=\varepsilon_{\mathrm{a}}=\varepsilon_{\mathrm{ti}}=\varepsilon_{\mathrm{vi}}=80, \kappa_{\mathrm{m}}=\kappa_{\mathrm{a}}=\kappa_{\mathrm{ti}}=\kappa_{\mathrm{vi}}=$ $1 \mathrm{~S} \mathrm{~m}^{-1}, \varepsilon_{\mathrm{vm}}=5$, and $\kappa_{\mathrm{vm}}=0 \mathrm{~S} \mathrm{~m}^{-1}$ for phase 'I'. In addition, the radii of individual cells and the overall aggregate are $R_{\mathrm{v}}=$ $5 \mu \mathrm{m}$, and $R=28 \mu \mathrm{m}$, respectively, while the membrane thickness of the individual cells in the model is $d_{\mathrm{vm}}=5 \mathrm{~nm}$. The thickness of the basement membrane shell surrounding the islet cluster is $d=50 \mathrm{~nm}$. For phase 'II', parameter values are as follows: $P=0.0585, P_{\mathrm{i}}=0.95, \varepsilon_{\mathrm{m}}=\varepsilon_{\mathrm{rm}}=\varepsilon_{\mathrm{a}}=\varepsilon_{\mathrm{i}}=\varepsilon_{\mathrm{vi}}=\varepsilon_{\mathrm{r}}=80$, $\kappa_{\mathrm{m}}=\kappa_{\mathrm{rm}}=\kappa_{\mathrm{a}}=\kappa_{\mathrm{i}}=\kappa_{\mathrm{vi}}=\kappa_{\mathrm{r}}=1 \mathrm{~S} \mathrm{~m}^{-1}, \varepsilon_{\mathrm{vm}}=5$, and $\kappa_{\mathrm{vm}}=0 \mathrm{~S} \mathrm{~m}^{-1}$. Finally, the radii of the individual cells, the inner lumen and 

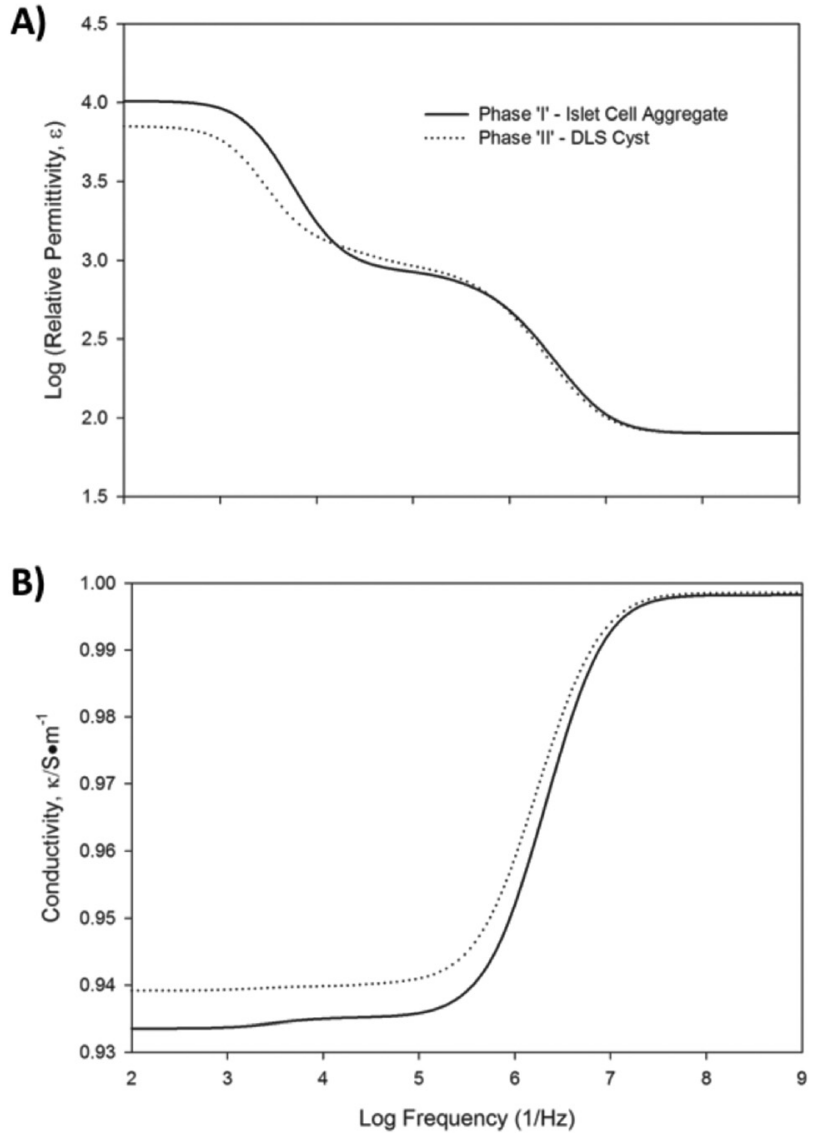

Fig. 3 FEA simulations showing (A) the relative permittivity and (B) conductivity of the islet differentiation process using the geometrical cell aggregate and cyst representing islets (phase ' $I$ ') and DLS (phase 'II'). The electrical parameters used in the FEA modeling are described in the text.

the overall cyst are $R_{\mathrm{v}}=5 \mu \mathrm{m}, R_{\mathrm{r}}=20 \mu \mathrm{m}$, and $R=28 \mu \mathrm{m}$, respectively; the membrane thicknesses of the cyst, the inner membrane surrounding lumen, and individual cells is $d_{\mathrm{vm}}=$ $5 \mathrm{~nm}$. The thicknesses of the basement membrane shell and the inner shell are $d=d_{\mathrm{rm}}=50 \mathrm{~nm}$. Dielectric parameters for permittivity and conductivity were obtained from values commonly used for FEA simulations of biological media. ${ }^{21}$

Fig. $3 \mathrm{~A}$ and $\mathrm{B}$ show the permittivity and conductivity spectra of the two phases. It is clear that the permittivity and conductivity spectra of phases 'I' and 'II' can be easily distinguishable from each other, exhibiting distinct relaxation spectra, containing two $\beta$ sub-dispersions. The low frequency (LF) sub-dispersion is caused by the presence of gap junctions connecting most cell membranes. This was shown to be attributed to charge density accumulation resulting from gap junctions as well as far-field interactions between closely adjacent faces. $^{30,31}$ The high frequency (HF) sub-dispersion on the other hand is caused by the inner cell mass of the islet. This is due to the high volume fraction occupied by the cell aggregates, and the respective resulting cell-cell interactions. The presence of very distinct and broad LF and HF sub-dispersions - resulting from gap junction charge density accumulation and cell-cell interactions, respectively - among larger, highly dense cell clusters is in contrast to smaller cellular epithelial aggregates where the minimal presence of gap junctions may not have a considerable contribution in the presence of the larger effects of tight junctions and interfacial interactions between adjacent cells as demonstrated before. ${ }^{20}$ It is also of interest to note that the effect of the lumen in phase 'II' on dielectric spectra is more significant than in past studies, largely due to the significantly larger size of islets and DLS compared to epithelial cystic differentiation. ${ }^{20}$

It is also important to note the LF and HF shifts in the dielectric spectra and sub-dispersion between the two phases, with the phase 'II' cystic DLS clearly showing a broadening shift in the HF dispersion indicative of the absence of an inner cell mass, signifying cell death and the presence of a lumen.

\section{Simplification of cell models for characterizing islet differentiation}

In order to examine the feasibility of analyzing the phases of islet differentiation (Fig. 1C and D) to cystic DLS structures, FEA simulations were conducted and compared to analytical values obtained for corresponding cell models (Fig. 1E and F). The results of this fitting analysis are presented in Fig. $4 \mathrm{~A}$ and B for phase 'I' (pancreatic islet cluster) and phase 'II' (DLS cyst), respectively. The islet cell cluster phase 'I' in Fig. 1C involves cellular aggregates that are connected by gap/tight junctions, thus forming a single shell - composed of basement membrane proteins - encompassing an area with a characteristic $\varepsilon_{\mathrm{i}}$ and $\kappa_{\mathrm{i}}$, representing the dielectric constants of the enclosed cellular aggregates (Fig. 1E). The parameters for the FEA modeling were the same as those used for "Phase 'I' Islet Cell Aggregate" in Fig. 3A and B. For the analytical model, the VI model employed in Fig. 1E was assumed using the equivalent parameters identical to those used in the FEA simulation. The outer shell of the model, representing the basement membrane surrounding islet clusters, was assumed to possess the same dielectric parameters as the intershell space and cell cytoplasms. As shown in our previous studies, the presence of a basement membrane with high conductivity as the surrounding media and cytoplasm does not elicit a distinct or significant dielectric dispersion response. ${ }^{20}$ An EMA of PS was used to describe the spherical shell enclosing the overall cell aggregate suspension, characterized by $\varepsilon_{\mathrm{i}}$ and $\kappa_{\mathrm{i}}$, obtained through an HAK EMA to describe the high volume fraction cell aggregate mixture. The results presented in Fig. 4A show an overlapping fit of the HF sub-dispersion using the VI analytical model presented. In terms of the error margin in permittivity and conductivity, the analytical model succeeds in characterizing the HF sub-dispersion, and provides a fairly accurate representation of the relaxation caused by the cell aggregates. Since the LF sub-dispersion is caused by the presence of a gap junction, it is thus not represented by the analytical cell model curve. Moreover, the slight deviation from the FEA simulations at the HF sub-dispersion is certainly attributed to the effects of the tight junctions, the complex cell geometry which does not equivalently translate to spherical structures, as well as the 
A)

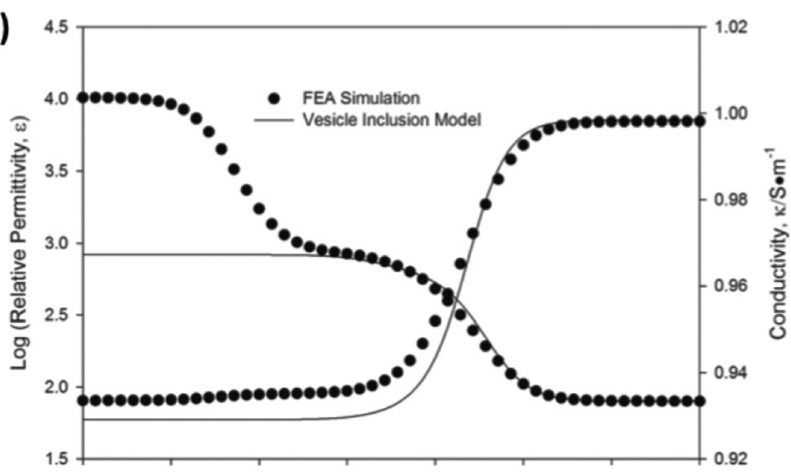

15 high volume fraction of the inner cell aggregates and the resulting cell membrane density, all of which are unaccounted for using the VI model and HAK/PS cell suspension EMA. However, we can therefore assume HAK/PS relations using the VI model to represent spherical cell aggregate suspensions with an inner cell mass connected through tight junctions.

In the case of the second phase 'II' of islet differentiation in Fig. 1D, the cystic DLS is simulated by FEA (Fig. 4B) with the same parameters used to generate 'Phase 'II' - DLS Cyst' in Fig. 3A and B. For the analytical model, the CC model in Fig. $1 \mathrm{~F}$ was assumed using the equivalent parameters identical to those used in the FEA simulation. An EMA of PS was used to describe the spherical shell enclosing the overall cell aggregate suspension, characterized by $\varepsilon_{\mathrm{i}}$ and $\kappa_{\mathrm{i}}$ values identical to islet cell aggregate phase 'I' in Fig. 4A, obtained through an HAK EMA used to describe the high volume fraction cell aggregate mixture in the intershell space. The simulation results shown in Fig. 4B, overlapped accordingly with the spectra obtained through the CC analytical model, with the similar slight deviations arising from similar factors to those described for Fig. 4A. We can therefore also assume that the inner cells and overall cyst suspensions of phase 'II' are well described using the CC analytical cell model and HAK/PS EMA for both conductivity and relative permittivity.

\section{Dielectric monitoring of islet differentiation}

Following electrode polarization correction and data fitting to the Cole-Cole equation (eqn (5)), the permittivity and conductivity of the islet-scaffold system were obtained. The dielectric spectra are shown for days 1 and 9 corresponding to the islet transformation stages from the islet to DLS (Fig. 5A) as it could be revealed by the corresponding light microscope images showing the islets and DLS embedded within the scaffold pores (Fig. 5B). Following a very similar trend to that observed in the FEA simulations of Fig. 3, the pancreatic islets and DLS displayed two visible sub-dispersions that were very

A)
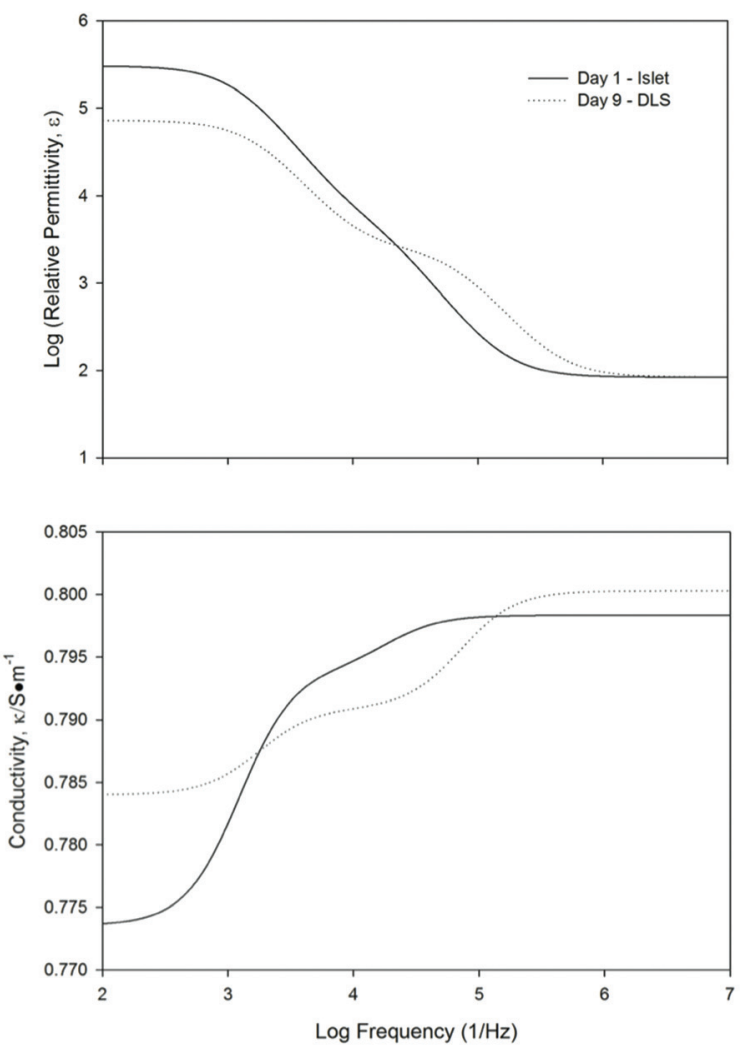

B)

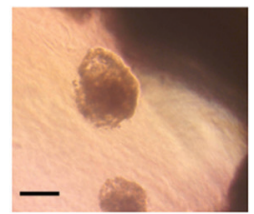

Day 1

C)

\begin{tabular}{c|c|c|c|c|c|}
\cline { 2 - 6 } & $\mathrm{f}_{\mathrm{cl}}(\mathrm{kHz})$ & $\mathrm{f}_{\mathrm{c} 2}(\mathrm{kHz})$ & $\Delta \varepsilon_{1}$ & $\Delta \varepsilon_{2}$ & $\kappa_{\mathrm{L}}\left(\mathrm{S} \cdot \mathrm{m}^{-1}\right)$ \\
\hline Day 1 (Islet) & 1.232 & 18.744 & 298901.637 & 4109.445 & 0.774 \\
\hline Day 9 (DLS) & 1.770 & 71.582 & 70193.618 & 2354.462 & 0.784 \\
\hline
\end{tabular}

Fig. 5 (A) Relative permittivity and conductivity of islet differentiation within the ECM gel-scaffold microenvironment in the bioreactor system. The cystic transformation of the islets into DLS is monitored and measurements are shown for days 1 and 9. (B) The corresponding light microscopy images showing the islet at day 1 and DLS at day 9 embedded within the ECM-gel scaffold (scale bar $=100 \mu \mathrm{m}$ ). (C) Table showing the dielectric parameters of islet and DLS phases obtained through Cole-Cole fitting of eqn (5). 
clearly distinguishable according to the respective capacitance and conductance measurements, with a lower permittivity shift in the LF dispersion and a broad permittivity shift in HF dispersion as the islets are continuously monitored through the differentiation process to DLS. The broadening of the HF dispersion from day 1 to 9 is characteristic of inner cell death that occurs with cyst formation. This finding correlates closely with the FEA analysis in Fig. 3, as well as proof-of-concept studies of epithelial cystic transformation. ${ }^{20}$

The findings from the permittivity and conductivity data for the cell-seeded scaffolds clearly show that the differences between the various structures are easily discernable. This is confirmed through the dielectric relaxation parameters obtained from fitting to the Cole-Cole relation (eqn (5)), given in Fig. 5C. The obtained dielectric parameters may be utilized as distinct 'cell signatures', representing phenotypic characteristics of a specific stage in islet differentiation to DLS. This may be further exploited to establish a controllable and easily monitored cellular process for islet regeneration, by potentially realizing the goal of regulating the differentiation of isolated human islets to proliferative DLS, followed by regeneration and re-differentiation into a large mass of ILS suitable for multiple transplantations.

Using the FEA tested analytical cell models in Fig. 4, it is possible to apply the Maxwell Wagner approximations for VI and CC models to determine parameters associated with islet and DLS phases, respectively. Firstly, as shown in Fig. 5C, the dielectric parameters for the $\beta$-dispersions are obtained by fitting the permittivity spectra into the Cole-Cole relation of eqn (5). The dielectric spectra of the islets within the ECM gelscaffold system is then used in the VI and CC models in order to obtain the corresponding permittivity of the islets and DLS, respectively, as well as those of the inner cell inclusions. As discussed earlier, only the HF sub-dispersion - resulting from cell aggregates joined by tight junctions - is taken into account, as LF sub-dispersions arising from gap junctions may not be represented by analytical cell models and the respective curve-fitting. Using confocal microscopy at the initial and end points of the experiment, an appropriate volume fraction, $P$, was obtained for both phases. This was determined to be $P=0.028$. Also, an inner volume fraction of cell aggregates, $P_{\mathrm{i}}=$ 0.95 , was assumed for both VI and CC models.

As outlined earlier, the outer shell in the $\mathrm{VI} / \mathrm{CC}$ and the inner shell of the CC model were assumed to represent the basement membrane; the dielectric parameter of which was assumed to be equivalent to the highly conductive suspending media, the intershell space between cells, as well as the cytoplasm of cells within the aggregate. The permittivity of the inclusions is then used to derive the values of the membrane capacitance and conductivity $-C_{\mathrm{vm}}$ and $\kappa_{\mathrm{vm}}$, respectively - of individual cells in the islet and DLS aggregates. These values were derived for the islet (day 1) and DLS (day 9) phases using the VI and CC models described by the Wagner approximation in Fig. 1E and $\mathrm{F}$ and eqn (6)-(14). Fig. 6A and B shows the correlation between the islet and DLS phases evaluated at days 1 and 9 fitted against spectra obtained using the VI and CC
A)

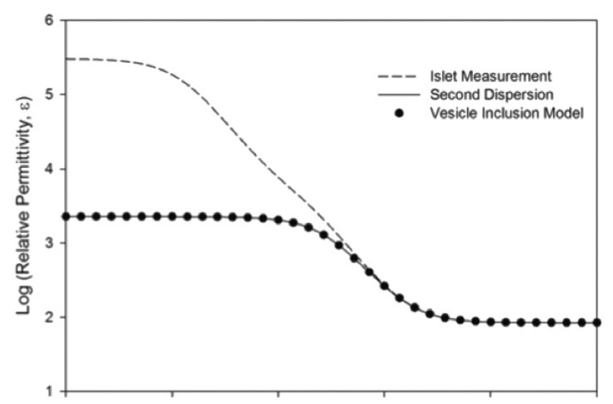

B)

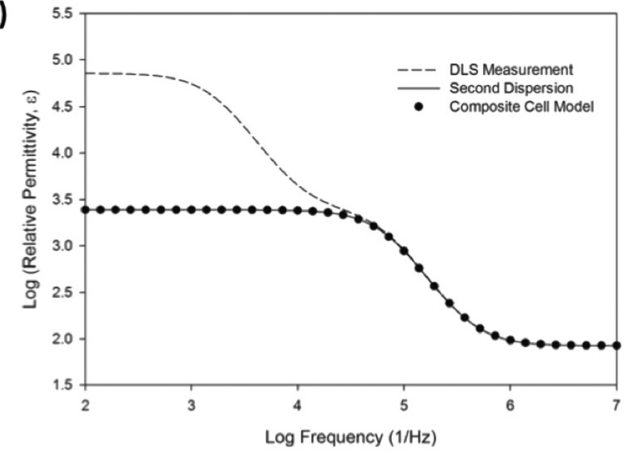

C)

\begin{tabular}{|c|c|c|}
\cline { 2 - 3 } \multicolumn{1}{c|}{} & $\mathrm{C}_{\mathrm{vm}}\left(\mu \mathrm{F} / \mathrm{cm}^{2}\right)$ & $\kappa_{\mathrm{vm}}(\mu \mathrm{S} / \mathrm{cm})$ \\
\hline Day 1 (Islet) & 3.54 & 26.53 \\
\hline Day 9(DLS) & 1.80 & 304.54 \\
\hline
\end{tabular}

Fig. 6 Fitting of the (A) day 1 islet and (B) day 9 DLS measurements to the analytical VI and CC models, respectively. (C) Table showing the obtained membrane capacitance and conductivity of the inner cells, $C_{v m}$ and $\kappa_{\mathrm{vm}}$, respectively, of the day 1 islet and day $9 \mathrm{DLS}$.

models, respectively. It is important to note the high correlation and low variance between the fitted and observed data for the cell permittivity and conductivity spectra at the HF subdispersion of interest. Fig. 6C shows the corresponding membrane capacitance values, which were calculated to obtain the fits in Fig. 6A and B.

It is interesting to note that the membrane capacitance of the islet structure differs markedly from that of the DLS, thus suggesting that the variation of electrical properties between islets and DLS cysts is directly correlated to the morphological and phenotypic shifts that occur as a result of differentiation. To better explain the decrease in membrane capacitance in biophysical terms from the islet to DLS individual cells, some parallels may be drawn with literature reports analyzing the electrical properties of neurons. This is due to shared characteristics in terms of electrical excitability and subsequent biochemical modulation. As mathematically described by the Hodgkin-Huxley cell membrane electric model, ${ }^{51}$ myelinated neurons possess a lower membrane capacitance due to the reduced number of ion channels, thus lowering the channel density, and/or increasing the spacing between channels. ${ }^{52-55}$ This is analogous to the reduced membrane capacitance observed as electrically actuated endocrine cells within the islets - containing a relatively high density of membrane bound ion channels required for hormone modulation - 
differentiate into epithelial cells comprising DLS. Further contribution to the decrease in membrane capacitance can be attributed to the increased level of actin filaments in the DLS cytoskeleton - required for maintaining phenotypic and morphological integrity. This relation has been demonstrated in a recent study through dielectrophoretic monitoring of epithelial cancer cell progression. ${ }^{56}$ Moreover, the associated increase in membrane conductivity may be attributed to a relatively higher activity of the less dense ion channels occurring in epithelial cells of the DLS. The activity of these ion channels is essential to the maintenance of a polarized cystic structure and associated osmotic homeostasis. ${ }^{57}$ This phenomenon has also been observed in a biophysical study of hepatocytes analyzing the conductance variation resulting from osmotic swelling. ${ }^{58}$

We must also note however the tentative nature of these values, since analytical cell models are currently not optimized to take into account LF sub-dispersions arising from gap junctions, as well as minor variations in HF sub-dispersions resulting from complex geometries as well as considerable cell-cell interactions caused by tight junctions and closely adjacent faces. Therefore, mathematical model optimization must be performed to arrive at more accurate reflections of cell membrane capacitance. However, despite the possible error in cell membrane calculations owing to the shortcomings of analytical models, this rapid method provides a very useful tool for analyzing and monitoring cellular differentiation processes within scaffold systems in a bioreactor setting, further providing a viable potential platform for observing, screening and regulating islet - and other tissues - differentiation, proliferation and regeneration.

Confocal microscopy was also employed in order to evaluate the islet to DLS to ILS transformation, in order to further confirm the viability of the transformation process. Fig. 7 shows the insulin and cytokeratin-19-marker content of the structures at days 1, 4 and 9. Insulin content provides insights
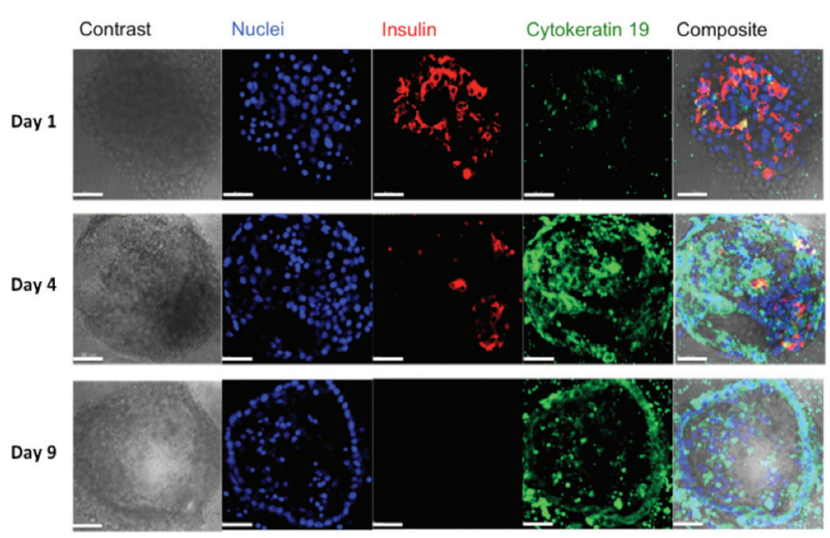

Fig. 7 Confocal microscopy images of the islet differentiation process showing islet at day 1 , a transition state at day 4 , and fully differentiated cystic DLS at day 9 (scale bar $=30 \mu \mathrm{m}$ ). The loss of insulin-positive cells demonstrates the loss of islet phenotype during the differentiation process, while the increase in cytokeratin 19-positive cells signifies the transformation to an epithelial phenotype characteristic of DLS. into the islet-like cyto-architecture of the structures, while CK19 is used as an epithelial cell marker used to identify the process of cystic transformation. These results complement those obtained with the permittivity measurements, in that the morphological characteristics directly correlate with the observed spectra.

\section{Conclusions}

In this study, we demonstrate a tissue bioreactor culture system, which allows for the culture and differentiation of human pancreatic islets embedded in an optimized ECM matrix within a geometrically controlled microfabricated scaffold. In addition, the cellular processes are monitored by a noninvasive method employing dielectric spectroscopy techniques. The results of these studies clearly demonstrate the efficacy of this approach, and the positive effects of all principles used in concert. The implementation of an online monitoring system not only provides accurate information concerning the morphological characteristics of cells and the associated volume fraction, but also provides valuable insights into the dielectric parameters that can be used as an additional tool for evaluating cellular behavior.

\section{Acknowledgements}

We gratefully thank the Natural Science and Engineering Research Council of Canada (NSERC), Canadian Institute of Health Research (CIHR)-Regenerative Medicine and Nanomedicine Program, JDRF (Juvenile Diabetes Research Foundation), and the Fonds Québécois de la recherche sur la Nature et les technologies (FQRNT) for their financial support. We would also like to thank Dr Koji Asami for all his scientific input and Mr Marius Cristian Pop for his expertise in CAD modeling of the biological cell cluster.

\section{Notes and references}

1 J. Daoud, L. Rosenberg and M. Tabrizian, Cell Transplant., 2010, 19, 1523-1535.

2 D. W. R. Gray, N. Titus and L. Badet, Expert Rev. Mol. Med., 2000, 2, 1-28.

3 J. Daoud, M. Petropavlovskaia, L. Rosenberg and M. Tabrizian, Biomaterials, 2010, 31, 1676-1682.

4 J. T. Daoud, M. S. Petropavlovskaia, J. M. Patapas, C. E. Degrandpre, R. W. Diraddo, L. Rosenberg and M. Tabrizian, Biomaterials, 2011, 32, 1536-1542.

5 L. P. Rutzky, S. Bilinski, M. Kloc, T. Phan, H. M. Zhang, S. M. Katz and S. M. Stepkowski, Transplantation, 2002, 74, 13-21.

6 J. A. Chromiak, J. Shansky, C. Perrone and H. H. Vandenburgh, In Vitro Cell. Dev. Biol.: Anim., 1998, 34, 694-703. 
7 L. Margolis, S. Hatfill, R. Chuaqui, C. Vocke, M. EmmertBuck, W. M. Linehan and P. H. Duray, J. Urol., 1999, 161, 290-297.

8 G. Vunjak-Novakovic, N. Searby, J. De Luis and L. E. Freed, Microgravity Transport Processes in Fluid, Thermal, Biological, and Materials Sciences, 2002, vol. 974, pp. 504-517.

9 M. A. Webb, S. L. Platton, A. R. Dennison and R. F. L. James, In Vitro Cell. Dev. Biol.: Anim., 2007, 43, 210214.

10 H. Blomeier, X. M. Zhang, C. Rives, M. Brissova, E. Hughes, M. Baker, A. C. Powers, D. B. Kaufman, L. D. Shea and W. L. Lowe, Transplantation, 2006, 82, 452459.

11 Y. M. Elcin, A. E. Elcin, R. G. Bretzel and T. Linn, Adv. Exp. Med. Biol., 2003, 534, 255-264.

12 D. M. Salvay, C. B. Rives, X. M. Zhang, F. Chen, D. B. Kaufman, W. L. Lowe and L. D. Shea, Transplantation, 2008, 85, 1456-1464.

13 J. C. Stendahl, D. B. Kaufman and S. I. Stupp, Cell Transplant., 2009, 18, 1-12.

14 Y. Wang, J. F. Lo, J. E. Mendoza-Elias, A. F. Adewola, T. A. Harvat, K. P. Kinzer, D. Lee, M. Qi, D. T. Eddington and J. Oberholzer, Bioanalysis, 2010, 2, 1729-1744.

15 A. F. Adewola, Y. Wang, T. Harvat, D. T. Eddington, D. Lee and J. Oberholzer, J. Visualized Exp., 2010.

16 A. F. Adewola, D. Lee, T. Harvat, J. Mohammed, D. T. Eddington, J. Oberholzer and Y. Wang, Biomed. Microdevices, 2010, 12, 409-417.

17 Y. Wang, J. Mohammed, T. Harvat, M. G. Qi, S. S. Wang, D. T. Eddington and J. Oberholzer, Am. J. Transplant., 2009, 9, 673-673.

18 J. F. Dishinger, K. R. Reid and R. T. Kennedy, Anal. Chem., 2009, 81, 3119-3127.

19 J. S. Mohammed, Y. Wang, T. A. Harvat, J. Oberholzer and D. T. Eddington, Lab Chip, 2009, 9, 97-106.

20 J. Daoud, K. Asami, L. Rosenberg and M. Tabrizian, Phys. Med. Biol., 2012, 57, 5097-5112.

21 K. Asami, J. Non-Cryst. Solids, 2002, 305, 268-277.

22 K. Asami, Prog. Polym. Sci., 2002, 27, 1617-1659.

23 T. Hanai, Colloid Polym. Sci., 1960, 171, 23-31.

24 H. P. Schwan, Adv. Biol. Med. Phys., 1957, 5, 147-209.

25 K. Asami, Bioelectrochemistry, 2013, 92, 14-21.

26 I. Ermolina, Y. Polevaya and Y. Feldman, Eur. Biophys. J., 2000, 29, 141-145.

27 P. O. Bagnaninchi and N. Drummond, Proc. Natl. Acad. Sci. U. S. A., 2011, 108, 6462-6467.

28 K. Asami and T. Yamaguchi, Biophys. J., 1992, 63, 14931499.

29 K. Asami and A. Irimajiri, Phys. Med. Biol., 2000, 45, 32853297.

30 E. Gheorghiu, C. Balut and M. Gheorghiu, Phys. Med. Biol., 2002, 47, 341-348.

31 K. Asami, J. Phys. D: Appl. Phys., 2007, 40, 3718-3727.

32 K. Asami and K. Sekine, J. Phys. D: Appl. Phys., 2007, 40, 1128-1133.
33 K. Asami and K. Sekine, J. Phys. D: Appl. Phys., 2007, 40, 2197-2204.

34 A. Ron, N. Fishelson, N. Croitoriu, D. Benayahu and Y. Shacham-Diamand, Biophys. Chem., 2009, 140, 39-50.

35 K. Asami, Jpn. J. Appl. Phys., 2010, 49, 127001.

36 A. M. Jamal, M. Lipsett, A. Hazrati, S. Paraskevas, D. Agapitos, D. Maysinger and L. Rosenberg, Cell Death Differ., 2003, 10, 987-996.

37 A. M. Jamal, M. Lipsett, R. Sladek, S. Laganiere, S. Hanley and L. Rosenberg, Cell Death Differ., 2005, 12, 702-712.

38 A. M. J. Shapiro, J. R. T. Lakey, E. A. Ryan, G. S. Korbutt, E. Toth, G. L. Warnock, N. M. Kneteman and R. V. Rajotte, N. Engl. J. Med., 2000, 343, 230-238.

39 K. Asami, Jpn. J. Appl. Phys., 2010, 49.

40 R. Landers, A. Pfister, U. Hubner, H. John, R. Schmelzeisen and R. Mulhaupt, J. Mater. Sci., 2002, 37, 3107-3116.

41 A. M. Yousefi, C. Gauvin, L. Sun, R. W. DiRaddo and J. Fernandes, Polym. Eng. Sci., 2007, 47, 608-618.

42 K. Asami and A. Irimajiri, Biochim. Biophys. Acta, Biomembr., 1984, 778, 570-578.

43 Y. Katsumoto, S. Omori, D. Yamamoto, A. Yasuda and K. Asami, Phys. Rev. E: Stat., Nonlinear, Soft Matter Phys., 2007, 75, 011911.

44 S. Omori, Y. Katsumoto, A. Yasuda and K. Asami, Phys. Rev. E: Stat., Nonlinear, Soft Matter Phys., 2006, 73, 050901.

45 G. F. Vunjak-Novakovic and M. Radisic, Methods Mol. Biol., 2004, 238, 131-146.

46 T. Hanai, K. Zhao, K. Asaka and K. Asami, Colloid Polym. Sci., 1993, 271, 766-773.

47 P. Baluk, S. Morikawa, A. Haskell, M. Mancuso and D. M. McDonald, Am. J. Pathol., 2003, 163, 1801-1815.

48 P. D. Yurchenco, Cold Spring Harbor Perspect. Biol., 2011, 3, a004911.

49 B. Xiang and S. K. Muthuswamy, Methods Enzymol., 2006, 406, 692-701.

50 R. Bertram and M. Pernarowski, Biophys. J., 74, 17221731.

51 A. L. Hodgkin and A. F. Huxley, J. Physiol., 1952, 117, 500544.

52 J. H. Caldwell, K. L. Schaller, R. S. Lasher, E. Peles and S. R. Levinson, Proc. Natl. Acad. Sci. U. S. A., 2000, 97, 56165620.

53 S. G. Waxman, Muscle Nerve, 1980, 3, 141-150.

54 K. V. Kolaric, G. Thomson, J. M. Edgar and A. M. Brown, Physiol. Rep., 2013, 1, e00059.

55 J. H. Caldwell and S. R. Levinson, in Advances in Molecular and Cell Biology, ed. A. M. Robert, Elsevier, 2004, vol. 32, pp. $15-50$.

56 A. Salmanzadeh, M. B. Sano, R. C. Gallo-Villanueva, P. C. Roberts, E. M. Schmelz and R. V. Davalos, Biomicrofluidics, 2013, 7, 011809.

57 H. W. Park and M. G. Lee, Cold Spring Harbor Perspect. Med., 2012, 2.

58 J. Graf, M. Rupnik, G. Zupancic and R. Zorec, Biophys. J., 1995, 68, 1359-1363. 